\title{
Ornstein-Zernike Theory for the Planar Random Surface Model
}

\author{
Thordur Jonsson
}

The Science Institute, University of Iceland, Dunhaga 3, 107 Reykjavik, Iceland

\begin{abstract}
We prove that the two-loop function in the planar random surface (PRS) model has Ornstein-Zernike decay for all noncritical values of the temperature. A notion of breathing is introduced and it is shown that surfaces do not breathe at noncritical temperatures. With the aid of a simple assumption, supported by mean field theory and numerical calculations, we prove that the scaling limit of the PRS-model exists and equals that of a free field.
\end{abstract}

\section{Introduction and Results}

Lattice gauge theories, string theory and various problems in statistical mechanics have recently stimulated interest in random surface theories, see [8-10] for review of some of the recent work. By now a considerable amount of information about a few models of lattice surfaces has been obtained. One of these is the model of SOStubes, studied by Abraham, Chayes and Chayes (ACC) [1-3]. Another one is the PRS-model studied by Eguchi, Kawai and Okamoto $[12,13]$ and in more detail by Durhuus, Fröhlich and Jónsson (DFJ) [4-7].

ACC have developed powerful methods, based on earlier work on the theory of classical fluids $[14,15]$, that enable them to analyze completely the noncritical behaviour of SOS-tubes. DFJ have calculated all the critical exponents in the PRSmodel [6] with the aid of a simple plausible hypothesis which is consistent with numerical simulations $[11,12]$ and mean field theory $[6,12]$. For other recent work on random surface models, see [10] and references therein.

The purpose of this paper is to show that the methods of ACC apply to the PRS-model. In particular, one can calculate power corrections to the exponential decay of the loop-loop correlation function and show that it has Ornstein-Zernike decay. Bricmont and Fröhlich have established Ornstein-Zernike decay for correlations in the self-avoiding surface model by different methods [16]. Furthermore, it is shown that the scaling limit of the two-loop function exists if the aforementioned hypothesis of [6] is true. The scaling limit equals that of simple random walk. This behaviour was strongly suggested by the results of [6]. Finally, 
we prove that typical surfaces contributing to the two-loop function of the PRSmodel are "thin" in a certain sense (absence of breathing) above the critical point. The absence of breathing persists at the critical point if the simple hypothesis of [6] is valid.

Before stating the results more precisely we establish our notation and recall, for the reader's convenience a few results from $[4,6]$. By a surface $S$ we mean a collection of plaquettes in $\mathbb{Z}^{d}$ some of whose edges are pairwise identified (glued together) in such a way that the result is a connected orientable two dimensional surface. The boundary of $S, \partial S$, is the collection of edges of plaquettes in $S$ that are not identified with any other edge. Sometimes the edges will be called links.

Let $\gamma_{1}, \gamma_{2}, \ldots, \gamma_{n}$ be loops in $\mathbb{Z}^{d}$. Denote by $\mathscr{S}\left(\gamma_{1}, \gamma_{2}, \ldots, \gamma_{n}\right)$ the family of all surfaces $S$ with planar topology (no handles) and $\partial S=\gamma_{1} \cup \gamma_{2} \cup \ldots \cup \gamma_{n}$. The $n$-loop function is defined by

$$
G_{\beta}\left(\gamma_{1}, \ldots, \gamma_{n}\right)=\sum_{S \in \mathscr{S}\left(\gamma_{1}, \ldots, \gamma_{n}\right)} e^{-\beta|S|},
$$

where $|S|$ is the number of plaquettes in $S$ counted with multiplicity. There exists a constant $\beta_{c}$, which depends only on $d$ such that all the loop functions are finite for $\beta>\beta_{c}$, but infinite for $\beta<\beta_{c}$. We call $\beta_{c}$ the critical point.

The mass $m(\beta)$ is defined by

$$
\lim _{a \rightarrow \infty} \frac{-1}{a} \ln G_{\beta}\left(\gamma, \gamma^{a}\right)=m(\beta)
$$

where $\gamma$ is a fixed loop and $\gamma^{a}$ its translate by a lattice spacings along a coordinate axis. In [4] the limit (1.2) is shown to exist. Furthermore, it is independent of $\gamma$, has the usual thermodynamic properties of mass (inverse correlation length) and is positive for $\beta>\beta_{c}$.

Let $\gamma_{L, M}$ be a square loop with side of length $L$ and $M$ lying in a coordinate plane. The potential $V_{\beta}(M)$ is defined by

$$
\lim _{L \rightarrow \infty} \frac{-1}{L} \ln G_{\beta}\left(\gamma_{L, M}\right)=V_{\beta}(M)
$$

and the string tension $\tau(\beta)$ by

$$
\tau(\beta)=\lim _{M \rightarrow \infty} \frac{1}{M} V_{\beta}(M) .
$$

These limits were shown to exist in [4]; they are positive and concave in $\beta$.

In Sects. 2 and 3 we prove the following two theorems.

Theorem 1.1. For any $\beta>\beta_{c}$ there is a constant $C_{\beta}>0$ such that

$$
G_{\beta}\left(\gamma, \gamma_{a}\right)=C_{\beta} \frac{e^{-m(\beta) a}}{a^{(d-1) / 2}}\left(1+O\left(\frac{1}{a}\right)\right)
$$

Theorem 1.2. For any $\beta>\beta_{c}$ and $L, M>0$ there exists $\varepsilon>0$ and a constant $C_{\beta}^{\prime}>0$ such that

$$
G_{\beta}\left(\gamma_{L, M}\right)=C_{\beta}^{\prime} e^{-L V_{\beta}(M)}\left(1+O\left(e^{-\varepsilon L}\right)\right)
$$


If $\lim m(\beta)=0$, one may try to construct the scaling limit of the two-loop function as follows: Choose $\beta(\lambda)$ such that $\beta(\lambda) \downarrow \beta_{c}$ as $\lambda \rightarrow \infty$ and $\lim _{\lambda \rightarrow \infty} \lambda m(\beta(\lambda))$ $=m_{*}>0$. Define

$$
G_{\lambda}^{*}(y)=G_{\beta(\lambda)}\left(\gamma, \gamma_{\lambda v}\right),
$$

where $y \in \mathbb{R}$ and $\lambda$ is chosen such that $\lambda y \in \mathbb{Z}$. If there is a function $\alpha(\lambda)$ such that

$$
\lim _{\lambda \rightarrow \infty} \alpha(\lambda)^{2} G_{\lambda}^{*}(y)=: G^{*}(y)
$$

exists in the sense of distributions for $y \neq 0$, and is nontrivial we call $G^{*}(y)$ the scaling limit of the two-loop function. It is for technical convenience that we choose to separate the loops in a coordinate direction. In general one can separate the loops in any direction and the scaling limit of the two-loop function is a function on $\mathbb{R}^{d}$, so $G^{*}(y)$ is in fact the scaling limit at $(0, \ldots, 0, y, 0, \ldots, 0)$. If $\alpha(\lambda)^{2}$ $\sim \lambda^{d-2+\eta}$, then $\eta$ is the anomalous dimension of the two point function of the continuum theory. Note that aside from a finite multiplicative factor, $G^{*}(y)$ is independent of $\gamma$. If, however, we want to scale up the size of the loops as $\lambda \rightarrow \infty$, we have to introduce loop-dependent renormalization unless $\tau(\beta) \sim m^{2}(\beta)$ as $\beta \rightarrow \beta_{c}$.

In the next section we introduce a modification of the PRS-model where surfaces are not allowed to contain certain "short intermediate loops," defined in Sect. 2. The modified model has a mass $m_{1}(\beta)$ and $m_{1}(\beta)>m(\beta)$ for $\beta>\beta_{c}$. In Sect. 4 we prove

Theorem 1.3. If $m_{1}\left(\beta_{c}\right)>m\left(\beta_{c}\right)=0$, then the scaling limit (1.8) exists and equals

$$
\text { const }\left(-\Delta+m_{*}^{2}\right)^{-1}(\xi)
$$

where $\xi=(0, \ldots, 0, y, 0, \ldots, 0)$ and $\Delta$ is the Laplacian on $\mathbb{R}^{d}$.

For a discussion of the meaning of the above theorem in terms of surfaces, see [6], where two distinct heuristic arguments were given for its validity. The hypothesis of Theorem (1.3) implies all the results on critical exponents in [6]. A new idea seems to be required in order to prove that $m_{1}\left(\beta_{c}\right)>m\left(\beta_{c}\right)=0$.

In Sect. 5 we introduce a simple criterion for breathing which amounts to the suppression of "short intermediate loops." We prove the absence of breathing whenever $m_{1}(\beta)>m(\beta)$.

\section{The Two-Loop Function}

In this section we study the two-loop function and prove Theorem 1.1. The formalism introduced here is also used in the subsequent sections.

If $\gamma$ is a loop, let $\gamma^{x}$ denote its translate by a lattice vector $x \in \mathbb{Z}^{d}$. The FourierLaplace transform of the two-loop function $G_{\beta}\left(\gamma, \gamma^{\prime}\right)$ is defined by

$$
\tilde{G}_{\beta}\left(\gamma, \gamma^{\prime} ; p\right)=\sum_{x \in \mathbb{Z}^{d}} e^{i p \cdot x} G_{\beta}\left(\gamma, \gamma^{\prime x}\right),
$$

whenever the sum is convergent, $p \in \mathbb{C}^{d}$. For $\beta>\beta_{c}$ the sum is absolutely convergent for all $p \in \mathbb{R}^{d}$. The inverse of the Fourier-Laplace transform is given by

$$
G_{\beta}\left(\gamma, \gamma^{\prime x}\right)=\frac{1}{(2 \pi)^{d}} \int e^{-i p \cdot x} \widetilde{G}_{\beta}\left(\gamma, \gamma^{\prime} ; p\right) d p,
$$

where the integration is over $[-\pi, \pi]^{d} \subset \mathbb{R}^{d}$. 
We recall the result

$$
G_{\beta}\left(\gamma, \gamma^{\prime x}\right) \leqq \text { const } e^{-m(\beta)\|x\|},
$$

proven in $[6$, Lemma B3], where

$$
\|x\|=\max _{1 \leqq i \leqq d}\left|x_{i}\right| .
$$

It follows that $\widetilde{G}_{\beta}\left(\gamma, \gamma^{\prime} ; p\right)$ is well-defined when $\beta>\beta_{c}$, provided the imaginary part of each $p_{i}, i=1, \ldots, d$, is sufficiently small.

Notation. If $k=\left(k_{1}, \ldots, k_{d}\right) \in \mathbb{C}^{d}$, we write $k=\left(k_{1}, \underline{k}\right)$, where $\underline{k}=\left(k_{2}, \ldots, k_{d}\right)$.

Lemma 2.1. For any $\beta>\beta_{c}$ and loops $\gamma, \gamma^{\prime}$, the function

$$
f\left(p_{1}\right)=\tilde{G}_{\beta}\left(\gamma, \gamma^{\prime} ;\left(p_{1}, \underline{0}\right)\right)
$$

is analytic in the strip $\left|\operatorname{Im} p_{1}\right|<m(\beta)$.

For any $p_{1} \in \mathbb{C}$ with $\left|\operatorname{Im} p_{1}\right|<m(\beta)$, the function

$$
\underline{p} \rightarrow \tilde{G}_{\beta}\left(\gamma, \gamma^{\prime} ;\left(p_{1}, \underline{p}\right)\right)
$$

in analytic on a neighbourhood of $\mathbb{R}^{d-1} \subset \mathbb{C}^{d-1}$.

Proof. The lemma follows from (2.3).

Notation. Let $S_{1}$ and $S_{2}$ be two surfaces with $\partial S_{1} \cap \partial S_{2} \neq \emptyset$. Let $l$ denote a nonempty collection of links in $\partial S_{1} \cap \partial S_{2}$. We denote by $S_{1} \coprod_{l} S_{2}$ the surface obtained by gluing $S_{1}$ to $S_{2}$ along the links in $l$. Note that $S_{1} \amalg_{l} S_{2}$ may not be uniquely determined if $l$ occurs more than once in $\partial S_{1} \cap \partial S_{2}$. This ambiguity will not cause any complications in the sequel.

Let $S$ be a surface. The edges of plaquettes in $S_{1}$ that do not belong to the boundary we call internal edges. An internal edge belongs to exactly two plaquettes in $S$. A loop $\gamma$ is an internal loop of $S$ if it is made of a connected sequence of internal edges. If $\gamma$ is an internal loop of $S$, we can "cut $S$ along $\gamma$ " (i.e. remove identifications of links in $\gamma$ ) and thereby obtain two surfaces $S_{1}$ and $S_{2}$ both of which contain $\gamma$ in their boundaries.

Let $\gamma_{1}$ be the loop that consists of two copies of the link joining the points $(0,0, \ldots, 0)$ and $(1,0, \ldots, 0)$. We define $\mathscr{S}_{1}\left(\gamma, \gamma^{\prime}\right)$ to be the collection of all surfaces $S \in \mathscr{S}\left(\gamma, \gamma^{\prime}\right)$ with the following two properties:

(i) There does not exist any translate $\gamma_{1}^{x}$ of $\gamma_{1}$ such that $\gamma_{1}^{x}$ is an internal loop of $S$ and if $S$ is cut along $\gamma_{1}^{x}$ then we obtain two surfaces $S_{1}$ and $S_{2}$ with $\partial S_{1}=\gamma \cup \gamma_{1}^{x}$ and $\partial S_{2}=\gamma_{1}^{x} \cup \gamma^{\prime}$.

(ii) It is not possible to decompose $S$

$$
S=S_{1} \coprod_{l} S_{2},
$$

where $S_{1} \in \mathscr{S}\left(\gamma, \gamma^{\prime}\right), l$ is a connected set of links in $\gamma^{\prime}, l \neq \gamma^{\prime}$, and $\partial S_{2}=\gamma_{l}$, where $\gamma_{l}$ is the loop obtained by going back and forth along the links in $l$.

Remark. The condition (ii) is for technical convenience only. In the terminology of [4] this condition amounts to the absence of pockets at $\gamma^{\prime}$. 
We call surfaces that satisfy condition (i) above irreducible and define the irreducible two-loop function by

$$
F_{\beta}\left(\gamma, \gamma^{\prime}\right)=\sum_{S \in \mathscr{\mathscr { S }}_{1}\left(\gamma, \gamma^{\prime}\right)} e^{-\beta|S|}
$$

Let $\mathscr{S}_{0}(\gamma, \gamma)$ be the collection of all surfaces in $\mathscr{S}\left(\gamma, \gamma^{\prime}\right)$ that have no pockets at $\gamma^{\prime}$,i.e. satisfy condition (ii) above. Define

$$
G_{\beta}^{\prime}\left(\gamma, \gamma^{\prime}\right)=\sum_{S \in \mathscr{S}_{0}\left(\gamma, \gamma^{\prime}\right)} e^{-\beta|S|}
$$

Lemma 2.2. Let e be a unit vector along a coordinate axis. Then, for any $\beta>\beta_{c}$,

$$
\lim _{a \rightarrow \infty} \frac{-1}{a} \ln G_{\beta}^{\prime}\left(\gamma, \gamma^{\prime a e}\right)=m(\beta)
$$

The limit

$$
\lim _{a \rightarrow \infty} \frac{-1}{a} \ln F_{\beta}\left(\gamma, \gamma^{\prime a e}\right):=m_{1}(\beta)
$$

exists and is a positive concave increasing function of $\beta$. Furthermore, $m_{1}(\beta)$ is independent of $\gamma, \gamma^{\prime}$ and

$$
m_{1}(\beta)>m(\beta)
$$

for all $\beta>\beta_{c}$.

Proof. The first statement is obvious because $G_{\beta}^{\prime}\left(\gamma, \gamma^{\prime a e}\right)$ and $G_{\beta}\left(\gamma, \gamma^{\prime a e}\right)$ differ by a finite multiple which is independent of $a$. The existence and properties of $m_{1}(\beta)$ are verified exactly as the corresponding results for $m(\beta)$ in [4]. The inequality (2.10) can be proven by the methods of [3].

Let us consider a surface $S \in \mathscr{S}_{0}\left(\gamma, \gamma^{\prime}\right)$. Either $S \in \mathscr{S}_{1}\left(\gamma, \gamma^{\prime}\right)$ or there exists a smallest subsurface $S_{1}$ of $S$ with $S_{1} \in \mathscr{S}_{1}\left(\gamma, \gamma_{1}^{x}\right)$ for some $x$ and

$$
S=S_{1} \coprod_{\gamma_{1}^{x}} S_{2},
$$

where $S_{2} \in \mathscr{S}_{0}\left(\gamma_{1}^{x}, \gamma^{\prime}\right)$. Note that $S_{1}$ and $S_{2}$ are uniquely determined by $S$. It follows that

$$
G_{\beta}^{\prime}\left(\gamma, \gamma^{\prime}\right)=F_{\beta}\left(\gamma, \gamma^{\prime}\right)+\sum_{x \in \mathbb{Z}^{d}} F_{\beta}\left(\gamma, \gamma_{1}^{x}\right) G_{\beta}^{\prime}\left(\gamma_{1}^{x}, \gamma^{\prime}\right)
$$

A convolution identity similar to (2.12) was one of the main technical tools in [6] as well as in $[1-3,14,15]$.

Fourier-Laplace transforming (2.12) we obtain

$$
\widetilde{G}_{\beta}^{\prime}\left(\gamma, \gamma^{\prime} ; p\right)=\tilde{F}_{\beta}\left(\gamma, \gamma^{\prime} ; p\right)+\tilde{F}_{\beta}\left(\gamma, \gamma_{1} ; p\right) \widetilde{G}_{\beta}^{\prime}\left(\gamma_{1}, \gamma^{\prime} ; p\right) .
$$

Taking $\gamma=\gamma^{\prime}=\gamma_{1},(2.13)$ becomes

$$
\widetilde{G}_{\beta}(p)=\frac{\tilde{F}_{\beta}(p)}{1-\widetilde{F}_{\beta}(p)},
$$

where

$$
G_{\beta}(p):=\tilde{G}_{\beta}^{\prime}\left(\gamma_{1}, \gamma_{1} ; p\right), \quad \tilde{F}_{\beta}(p):=\tilde{F}_{\beta}\left(\gamma_{1}, \gamma_{1} ; p\right) .
$$


Let $e$ be a unit vector along the first coordinate axis. Define

$$
G_{\beta}(a):=G_{\beta}^{\prime}\left(\gamma_{1}, \gamma_{1}^{a e}\right),
$$

where $a \in \mathbb{Z}^{+}$. Inverting (2.14) we have

$$
G_{\beta}(a)=\frac{1}{(2 \pi)^{d}} \int e^{-i p_{1} a} \frac{\widetilde{F}_{\beta}(p)}{1-\widetilde{F}_{\beta}(p)} d p .
$$

The remainder of this section is concerned with an analysis of the integral (2.17) along the lines of [2]. We fix a value of $\beta>\beta_{c}$ so $m_{1}(\beta)>m(\beta)>0$ and divide the region of integration in (2.17) in two parts: $|\underline{p}| \geqq \delta$ and $|\underline{p}|<\delta$. As $a \rightarrow \infty$, we will show that the contribution from the first region is exponentially small relative to the second one. For $|p|<\delta$ the dominant contribution comes from a pole in the complex $p_{1}$-plane and this leads to Theorem 1 .

Lemma 2.3. The function

$$
h\left(p_{1}\right)=\widetilde{G}_{\beta}\left(\left(p_{1}, \underline{0}\right)\right)
$$

has simple poles at $p_{1}= \pm i m(\beta)$ and extends meromorphically to the larger region $\left|\operatorname{Im} p_{1}\right|<m_{1}(\beta)$.

Proof. This follows from the subadditivity bounds

$$
\begin{gathered}
G_{\beta}(a) \leqq \text { const } e^{-m(\beta) a} \\
F_{\beta}\left(\gamma_{1}, \gamma_{1}^{a e}\right) \leqq \text { const } e^{-m_{1}(\beta) a}
\end{gathered}
$$

and (2.14).

Lemma 2.4. For any $\delta>0$, there exist $\varepsilon>0$ and $\varepsilon^{\prime}>0$, such that

$$
\left|1-\widetilde{F}_{\beta}\left(\left(p_{1}, \underline{p}\right)\right)\right|>\varepsilon^{\prime},
$$

whenever $|\underline{p}|>\delta$ and $\left|\operatorname{Im} p_{1}\right|<m(\beta)+\varepsilon$.

Proof. The proof is very similar to the proof of Lemma B.1 in [2] so we omit it.

Lemma 2.5. Given $\delta>0$, there exists $\varepsilon^{\prime \prime}>0$ and a constant $C$ such that

$$
\left|\int_{-\pi}^{\pi} d p_{1} \int_{|\underline{p}|>\delta} d \underline{\underline{G}} \tilde{G}_{\beta}(p) e^{-i p_{1} a}\right| \leqq C e^{-\left(m(\beta)+\varepsilon^{\prime \prime}\right) a}
$$

for any $a \in \mathbb{Z}^{+}$.

Proof. By Lemma 2.4 there is an $\varepsilon^{\prime \prime}>0$ such that $2 \varepsilon^{\prime \prime}+m(\beta)<m_{1}(\beta)$ and the integrand in (2.21) is analytic in $p_{1}$ in the region $\left|\operatorname{Im} p_{1}\right|<m(\beta)+2 \varepsilon^{\prime \prime}$. We can therefore move the $p_{1}$-integration to the line segment in the complex $p_{1}$-plane connecting $-\pi-i\left(m(\beta)+\varepsilon^{\prime \prime}\right)$ and $\pi-i\left(m(\beta)+\varepsilon^{\prime \prime}\right)$. Thus, the left-hand side of $(2.21)$ equals

$$
\left|e^{-\left(m(\beta)+\varepsilon^{\prime \prime}\right) a} \int_{-\pi}^{\pi} d p_{1} \int_{|\underline{p}|>\delta} d \underline{\underline{G}} \widetilde{G}_{\beta}\left(\left(p_{1}-i\left(m(\beta)+\varepsilon^{\prime \prime}\right), \underline{p}\right)\right) e^{-i p_{1} a}\right| \leqq \text { const } e^{-\left(m(\beta)+\varepsilon^{\prime \prime}\right) a} .
$$


Lemma 2.6. If $\delta>0$ is sufficiently small, and $|p|<\delta$, there is $\mu \in\left(m(\beta), m_{1}(\beta)\right)$, such that $1-F\left(\left(p_{1}, p\right)\right)$ has only one simple zero as a function of $p_{1}$ in the region $-\mu$ $<\operatorname{Im} p_{1}<0$. This zero is a real analytic function of $\underline{p}$.

Proof. The lemma follows from Lemma 2.3, Rouché's Theorem and the analytic implicit function theorem in the same way as in [2, Lemma B.2].

Proof of Theorem 1.1. By Lemma 2.5 it suffices to show that

$$
g(a):=\int_{-\pi}^{\pi} d p_{1} \int_{|\underline{p}|<\delta} d \underline{p} \widetilde{G}_{\beta}\left(\left(p_{1}, \underline{p}\right)\right) e^{-i p_{1} a}
$$

has the desired asymptotic decay when $\delta>0$ is sufficiently small. Choose $\delta$ so that Lemma 2.6 holds. Let $-i M(\underline{p})$ be the pole of $\widetilde{G}_{\beta}\left(\left(p_{1}, p\right)\right)$ in the region $-\mu<\operatorname{Im} p_{1}<0$. Looking at the explicit formula for $\tilde{F}_{\beta}\left(\left(p_{1}, \underline{p}\right)\right)$ it is easy to see that

$$
M(\underline{p})=m(\beta)+\alpha \underline{p}^{2}+O\left(\underline{p}^{4}\right),
$$

where $\alpha>0$.

To evaluate $g(a)$ we integrate the function

$$
f\left(p_{1}\right):=\int_{|\underline{p}|<\delta} d \underline{p} e^{-i p_{1} a} G_{\beta}\left(\left(p_{1}, \underline{p}\right)\right)
$$

along a contour $\Gamma$ which is defined to be the boundary of the rectangle

$$
\{z \in \mathbb{C} \mid-\pi<\operatorname{Re} z<\pi, 0>\operatorname{Im} z>-\mu\} .
$$

The contribution to

$$
\int_{\Gamma} f\left(p_{1}\right) d p_{1}
$$

coming from the sides $\operatorname{Re} p_{1}=-\pi$ and $\operatorname{Re} p_{1}=\pi$ cancel. The contribution from the side with $\operatorname{Im} p_{1}=-\mu$ is $O\left(e^{-a \mu}\right)$ so $g(a)$ is essentially given by the residue at the simple pole. Hence, up to exponentially small corrections,

$$
g(a)=\int_{|\underline{\mid}|<\delta} h(\underline{p}) e^{-a M(\underline{p})} d \underline{p},
$$

where $h(\underline{p})$ is a bounded continuous function independent of $a$. Making use of (2.24), the desired result

$$
g(a)=\mathrm{const} \frac{e^{-m(\beta) a}}{a^{(d-1) / 2}}\left(1+O\left(\frac{1}{a}\right)\right)
$$

follows easily.

\section{The One Loop-Function}

In this section we prove Theorem 1.2. As in Sect. 2 the essential step is the derivation of a convolution identity.

Let $\mathscr{S}^{\prime}\left(\gamma_{L, M}\right)$ be the collection of all surfaces $S \in \mathscr{S}\left(\gamma_{L, M}\right)$ with the following property: The plaquettes adjacent to one of the $M$-sides, the left one say, lie in the interior of the loop $\gamma_{L, M}$ in its plane. We clearly have

$$
G^{\prime}\left(\gamma_{L, M}\right):=\sum_{S \in \mathscr{S}^{\prime}\left(\gamma_{L, M}\right)} e^{-\beta|S|}=e^{-\beta M} G\left(\gamma_{L-1, M}\right) .
$$


Let $\mathscr{S}^{\prime \prime}\left(\gamma_{L, M}\right)$ be the subset of $\mathscr{S}^{\prime}\left(\gamma_{L, M}\right)$ consisting of those surfaces that cannot be decomposed into two surfaces $S_{1} \in \mathscr{S}^{\prime}\left(\gamma_{L_{1}, M}\right), S_{2} \in \mathscr{S}^{\prime}\left(\gamma_{L_{2}, M}\right)$ with $L_{1}+L_{2}=L$ by cutting along $M$ internal links. Defining

$$
G_{\beta}^{\prime \prime}\left(\gamma_{L, M}\right):=\sum_{S \in \mathscr{S}^{\prime \prime}\left(\gamma_{L}, M\right)} e^{-\beta|S|},
$$

we have, for $L \geqq 2$,

$$
G_{\beta}^{\prime}\left(\gamma_{L, M}\right)=G_{\beta}^{\prime \prime}\left(\gamma_{L, M}\right)+\sum_{N=1}^{L-1} G_{\beta}^{\prime \prime}\left(\gamma_{N, M}\right) G_{\beta}^{\prime}\left(\gamma_{L-N, M}\right)
$$

We define the Laplace-transform of $G_{\beta}^{\prime}\left(\gamma_{L, M}\right)$ by

$$
\hat{G}_{\beta}^{\prime}(z):=\sum_{L=1}^{\infty} z^{L} G_{\beta}^{\prime}\left(\gamma_{L, M}\right)
$$

$z \in \mathbb{C}$. The Laplace-transform $\hat{G}_{\beta}^{\prime \prime}(z)$ is defined by an analogous formula. Clearly $\hat{G}_{\beta}^{\prime}(z)$ and $\hat{G}_{\beta}^{\prime \prime}(z)$ are well defined and analytic for $|z|<V_{\beta}(M)$ and $|z|<V_{\beta}^{\prime \prime}(M)$, respectively, where

$$
V_{\beta}^{\prime \prime}(M)=\lim _{L \rightarrow \infty} \frac{-1}{L} \ln G_{\beta}^{\prime \prime}\left(\gamma_{L, M}\right)
$$

The limit (3.5) exists and is finite by standard arguments for any $\beta>\beta_{c}$ [4]. Laplace-transforming (3.3) we obtain

$$
\hat{G}^{\prime}(z)=\frac{\hat{G}_{\beta}^{\prime \prime}(z)}{1-\hat{G}_{\beta}^{\prime \prime}(z)} .
$$

By an adaptation the methods developed in [3] one can show that $V_{\beta}^{\prime \prime}(M)>V_{\beta}(M)$ whenever $\beta>\beta_{c}$. Equation (3.6) therefore gives a meromorphic extension of $\hat{G}_{\beta}^{\prime}(z)$. The proof can now be completed as in [2, Theorem 2.4].

\section{The Scaling Limit}

In this section we prove Theorem 1.3. For convenience we take $\gamma=\gamma_{1}$ in the scaled two-loop function $G_{\lambda}^{*}(y)$ defined in (1.7). Then, separating the loops in the first coordinate direction and using (2.17),

$$
\begin{aligned}
G_{\lambda}^{*}(y) & =\frac{1}{(2 \pi)^{d}} \int e^{-i p_{1} \lambda y} \frac{\widetilde{F}_{\beta}(p)}{1-\tilde{F}_{\beta}(p)} d p \\
& =\frac{1}{(2 \pi \lambda)^{d}} \int_{-\lambda \pi}^{\lambda \pi} d p_{1} \ldots \int_{-\lambda \pi}^{\lambda \pi} d p_{d} e^{-i p_{1} y} \frac{\widetilde{F}_{\beta}\left(\lambda^{-1} p\right)}{1-\widetilde{F}_{\beta}\left(\lambda^{-1} p\right)},
\end{aligned}
$$

where $\beta=\beta(\lambda)$ is chosen such that $\lambda m(\beta(\lambda)) \rightarrow m_{*}>0$ as $\lambda \rightarrow \infty$.

Now note that $\widetilde{F}_{\beta}(p)$ is uniformly bounded for $\beta \geqq \beta_{c}$ and real analytic, so by the calculations in Sect. 2 [cf. Eq. (2.24)],

$$
1-\widetilde{F}_{\beta}\left(\lambda^{-1} p\right)=k_{1}(\beta)\left(m^{2}(\beta)+\lambda^{-2} p_{1}^{2}\right)+k_{2}(\beta) \hat{\lambda}^{-2} \underline{p}^{2}+O\left(\lambda^{-4}\right),
$$


where $k_{1}$ and $k_{2}$ are strictly positive functions of $\beta$ in the region $\beta \geqq \beta_{c}$. Hence,

$$
\begin{aligned}
\lambda^{d-2} G_{\lambda}^{*}(y)= & \frac{1}{(2 \pi)^{d}} \int_{-\lambda \pi}^{\lambda \pi} d p_{1} \ldots \int_{-\lambda \pi}^{\lambda \pi} d p_{d} e^{-i p_{1} y} \widetilde{F}_{\beta}\left(\lambda^{-1} p\right) \\
& \times\left[k_{1}(\beta)\left(\lambda^{2} m^{2}(\beta)+p_{1}^{2}\right)+k_{2}(\beta) \underline{p}^{2}+O\left(\lambda^{-2}\right)\right]^{-1} .
\end{aligned}
$$

It is straightforward to verify that the limit of (4.3) exists (as a distribution in $y \neq 0$ ) as $\lambda \rightarrow \infty$ and equals

$$
\text { const } \int_{\mathbb{R}^{d}} e^{-i p_{1} y}\left(m_{*}^{2}+p^{2}\right)^{-1} d p=\text { const }\left(-\Delta+m_{*}^{2}\right)^{-1}(y, 0, \ldots, 0),
$$

where the constant is positive and depends on $k_{1}\left(\beta_{c}\right)$ and $k_{2}\left(\beta_{c}\right)$. This completes the proof.

\section{Absence of Breathing}

Let $\mathscr{S}^{n}\left(\gamma_{1}, \gamma_{1}^{x}\right)$ be the collection of all surfaces $S$ in $\mathscr{S}_{0}\left(\gamma_{1}, \gamma_{1}^{x}\right)$ that can be decomposed into exactly $n$ irreducible surfaces:

$$
S=S_{1} \coprod_{\gamma_{1}^{x}} S_{2} \amalg_{\gamma_{1}^{x_{2}}} \ldots \amalg_{\gamma_{1}^{x_{n}-1}} S_{n}
$$

for some $x_{1}, \ldots, x_{n-1} \in \mathbb{Z}^{d}$. Define

$$
G_{\beta}^{n}\left(\gamma_{1}, \gamma_{1}^{x}\right)=\sum_{S \in \mathscr{S}^{n}\left(\gamma_{1}, \gamma_{1}^{x}\right)} e^{-\beta|S|}
$$

and

$$
\varrho_{x}(\beta)=\left(|x| G_{\beta}^{\prime}\left(\gamma_{1}, \gamma_{1}^{x}\right)\right)^{-1} \sum_{n=0}^{\infty} n G_{\beta}^{n}\left(\gamma_{1}, \gamma_{1}^{x}\right) .
$$

The quantity $\varrho_{x}(\beta)$ has an interpretation as the average density of translates of $\gamma_{1}$ along surfaces with boundary $\gamma_{1} \cup \gamma_{1}^{x}$.

Theorem 5.1. If $x \rightarrow \infty$ in a coordinate direction, then the limit

$$
\varrho(\beta)=\lim _{x \rightarrow \infty} \varrho_{x}(\beta)
$$

exists and is positive for any $\beta>\beta_{c}$.

Remark. The above theorem means that surfaces are thin in a certain sense for $\beta>\beta_{c}$. If $\varrho(\beta)$ were equal to 0 , we would say that the surfaces breathe. This notion of breathing is different from the ones introduced in $[2,7]$ but we find the simplicity of this one appealing and expect it to be equivalent to the one introduced by ACC, at least for the PRS-model. A notion of breathing called "restricted breathing," similar to the one used here is briefly discussed in [3].

Proof. We observe that

$$
G_{\beta}^{n}\left(\gamma_{1}, \gamma_{1}^{x}\right)=\sum_{x_{1}, \ldots, x_{n}} F_{\beta}\left(\gamma_{1}, \gamma_{1}^{x_{1}}\right) F_{\beta}\left(\gamma_{1}^{x_{1}}, \gamma_{1}^{x_{2}}\right) \ldots F_{\beta}\left(\gamma_{1}^{x_{n}}, \gamma_{1}^{x}\right)
$$

so the Fourier-Laplace transform of $G_{\beta}^{n}$ is given by

$$
\widetilde{G}_{\beta}^{n}\left(\gamma_{1}, \gamma_{1} ; p\right)=\left(\widetilde{F}_{\beta}(p)\right)^{n},
$$


with $\tilde{F}_{\beta}(p)$ as in Eq. (2.15). Hence,

$$
\begin{aligned}
\sum_{n=0}^{\infty} n G_{\beta}^{n}\left(\gamma_{1}, \gamma_{1}^{x}\right) & =\int e^{-i p \cdot x} \sum_{n=0}^{\infty} n\left(\widetilde{F}_{\beta}(p)\right)^{n} d p \\
& =\int e^{-i p \cdot x} \frac{\tilde{F}_{\beta}(p)}{\left(1-\widetilde{F}_{\beta}(p)\right)^{2}} d p
\end{aligned}
$$

One can mimick the analysis of Sect. 2 to show that for $\beta>\beta_{c}$,

$$
\int e^{-i p_{1} a} \frac{\tilde{F}_{\beta}(p)}{\left(1-\widetilde{F}_{\beta}(p)\right)^{2}}=\frac{\text { const } e^{-m(\beta) a}}{a^{1 / 2(d-3)}}\left(1+O\left(\frac{1}{a}\right)\right),
$$

where the constant is positive. The only difference is that now there is a double pole in $p_{1}$, which changes the power of $a$ by 1 . The desired result follows now from Theorem 1.

Remark. If $m_{1}\left(\beta_{c}\right)>m\left(\beta_{c}\right)=0$, then it is easily verified, making use of Eq. (5.5), that $\varrho_{x}\left(\beta_{c}\right)=\infty$ for $d=2,3,4$ (due to infrared divergences) and $\varrho\left(\beta_{c}\right)>0$ for $d \geqq 5$.

\section{Concluding Remarks}

In this paper the results of [6] have been corroborated, There is still one important piece missing in the complete solution of the PRS-model, namely to show that $m_{1}\left(\beta_{c}\right)>m\left(\beta_{c}\right)=0$. For this purpose it would suffice to prove that the susceptibility $\tilde{F}_{\beta}(0)$ is finite and greater than 1 for some $\beta<\beta_{c}$. In principle this could be done on a computer.

In a recent very interesting paper [17] Baumann and Berg present the results of a MC simulation of a modified planar random surface model, where any two plaquettes that are glued together are not allowed to overlap. This model is more amenable to an efficient simulation than the original PRS-model is. Baumann and Berg find the critical exponents $\gamma=1 / 4$ and $\eta=1$ with an accuracy of almost $10 \%$. This result is rather surprising because naively one would not expect that a local constraint could change the critical behaviour. As explained in [17] the methods of [6] (and the present paper) do not apply directly to the modified PRS-model. There is some hope that a suitable modification of those methods will work.

Acknowledgements. I would like to thank B. Durhuus for helpful discussions and Nordita for hospitality.

\section{References}

1. Abraham, D.B., Chayes, J.T., Chayes, L.: Statistical mechanics of lattice tubes. Phys. Rev. D 30, 841 (1984)

2. Abraham, D.B., Chayes, J.T., Chayes, L.: Random surface correlation functions. Commun. Math. Phys. 96, 439 (1984)

3. Abraham, D.B., Chayes, J.T., Chayes, L.: Nonperturbative analysis of a model of random surfaces. Nucl. Phys. B 251 [FS13], 553 (1985)

4. Durhuus, B., Fröhlich, J., Jonsson, T.: Self-avoiding and planar random surfaces on the lattice. Nucl. Phys. B 225 [FS9], 185 (1983) 
5. Durhuus, B., Fröhlich, J., Jonsson, T.: Critical properties of a model of planar random surfaces. Phys. Lett. 137 B, 93 (1984)

6. Durhuus, D., Fröhlich, J., Jonsson, T.: Critical behaviour in a model of planar random surfaces. Nucl. Phys. B 240 [FS12], 453 (1984)

7. Durhuus, B., Fröhlich, J., Jonsson, T.: Reflection positivity and tree inequalities in a theory of planar random surfaces. Nucl. Phys. B 257 [FS14], 779 (1985)

8. Fröhlich, J.: The statistical mechanics of surfaces, in Applications of field theory to statistical mechanics. Lecture Notes in Physics Vol. 216. Garrido, L. (ed.) Berlin, Heidelberg, New York: Springer 1985

9. Chayes, J., Chayes, L.: Percolation and random media. Les Houches lectures 1984

10. Fröhlich, J.: Survey of random surface theory. In: Recent developments in quantum field theory. Ambjörn, J., Durhuus, B.J., Petersen, J.L. (eds.). Amsterdam: North-Holland 1985

11. Berg, B., Billoire, B.: A Monte Carlo simulation of random surfaces. Phys. Lett. 139 B, 297 (1984)

12. Kawai, H., Okamoto, Y.: Entropy of planar random surfaces on the lattice. Phys. Lett. $130 \mathrm{~B}$, 415 (1983)

13. Eguchi, T., Kawai, H.: The number of random surfaces on the lattice and large $-N$ gauge theory. Phys. Lett. 110 B, 143; (1982); Planar random surfaces on the lattice. Phys. Lett. 114 B, 247 (1982)

14. Ornstein, L.S., Zernike, F.: Accidental deviations of density and opalescence at the critical point of a single substance. Proc. Acad. Sci. Amsterdam 17, 793 (1915)

15. Abraham, D.B., Kunz, H.: Ornstein-Zernike theory of classical fluids at low density. Phys. Rev. Lett. 39, 1011 (1977)

16. Bricmont, J., Fröhlich, J.: Statistical mechanical methods in particle structure analysis of lattice field theories, I. General theory. Nucl. Phys. B 251 [FS13], 517 (1985); II. Scalar and surface models. Commun. Math. Phys. 98, 553 (1985)

17. Baumann, B., Berg, B.: Non-trivial lattice random surfaces. Phys. Lett. 164 B, 131 (1985)

Communicated by A. Jaffe

Received February 7, 1986; in revised form April 7, 1986 
